\title{
Carnets
}

Revue électronique d'études françaises de l'APEF

Première Série - 1 Numéro Spécial | 2009

Cultures littéraires : nouvelles performances \& développement

\section{Statut de la lecture et du lecteur dans la culture contemporaine. Les nouveaux protocoles}

José Domingues de Almeida

\section{(2) OpenEdition}

Journals

Édition électronique

URL : http://journals.openedition.org/carnets/3998

DOI : 10.4000/carnets.3998

ISSN : 1646-7698

Éditeur

APEF

Édition imprimée

Date de publication : 1 juin 2009

Pagination : 281-288

Référence électronique

José Domingues de Almeida, «Statut de la lecture et du lecteur dans la culture contemporaine. Les nouveaux protocoles », Carnets [En ligne], Première Série - 1 Numéro Spécial | 2009, mis en ligne le 16 juin 2018, consulté le 02 mai 2019. URL : http://journals.openedition.org/carnets/3998 ; DOI : $10.4000 /$ carnets.3998 


\title{
STATUT DE LA LECTURE ET DU LECTEUR DANS LA CULTURE CONTEMPORAINE \\ Les nouveaux protocoles
}

José DOMINGUES DE ALMEIDA

Université de Porto

jalmeida@letras.up.pt

\begin{abstract}
Résumé
L'approche de la contemporanéité littéraire s'est souvent effectuée du côté du texte et/ou de l'instance auctorale, mais elle laisse entrevoir d'importantes mutations opérées également du côté du lecteur qu'un essai-roman tel que Comme un roman de Daniel Pennac est venu éclairer en 1992, et que d'autres apports critiques viendront confirmer. Nous nous proposons de passer en revue ces nouvelles complexités du champ littéraire contemporain et de les systématiser en tenant compte des apports de la critique et de la théorie de ces dernières années; lesquelles se sont penchées sur le phénomène de la réception du texte littéraire dans le cadre de l'utilité (futilité) de la culture littéraire à notre époque (Ancion, Eco, Compagnon, etc.). II s'agira en tous cas d'interroger la culture littéraire contemporaine par rapport à la valeur qu'elle assigne à l'acte d'écrire.
\end{abstract}

\section{Abstract}

The approach of literary contemporaneity has often suggested from the text or the author's point of view, but the reader's point of view has been more and more emphasised, for example in the essaynovel Comme un roman by Daniel Pennac in 1992, confirmed by more recent criticism and literary studies (Ancion, Eco, Compagnon, etc.). So, reading must be questioned in the global context of contemporary literary culture as well.

Mots-clés: Lecture, Littérature, Contemporain, Postmoderne, Protocole

Keywords: Reading, Literature, Contemporaneity, Postmodern, Protocole 
Thierry Guichard rappelle dans un dossier de Prétexte consacré à l'état de la prose contemporaine ce qui peut sembler une évidence : la littérature est la rencontre de "deux besoins, deux exigences : écrire et lire“ (Guichard, 1995: 45). La tradition littéraire n’a, pendant longtemps, interrogé que la prévalence de l'instance auctorale, avant de la proscrire au profit de l'autonomie du texte. Le rôle et la qualité du lecteur n'ont dès lors cessé d'osciller entre intrusion, abstraction ou perfection, selon que prévalait l'historicisme ou le formalisme (Compagnon, 1998:150-156).

L'émergence avérée d'un tournant dans la fiction française à partir de la fin des années septante, associée à la déroute des théories littéraires axées sur la "textualité", redéfinit le rôle assigné au lecteur par la contemporanéité littéraire.

De fait, la tentative de confrontation des théories littéraires au sens commun entreprise par Antoine Compagnon permet de reconsidérer et de faire le point sur quelques truismes que la théorie avait habilement escamotés. Elle opère sur la réception du texte littéraire une synthèse dialectique parallèle à celle que l'on a constatée pour la production littéraire contemporaine.

Ce faisant, la question du "lecteur" répond implicitement à deux interrogations du moment : rétablir un rôle équilibré du lecteur dont on aperçoit la "résistance" malgré les coups portés par le "terrorisme" des théories, et éclairer sa nouvelle posture face aux caractéristiques du roman contemporain.

Compagnon passe en revue les aléas du lecteur pris au piège de la théorie littéraire structuraliste, hantée par la description d'un texte marqué par la neutralité et l'autonomie. Ce théoricien regrette que le lecteur y soit réduit à la condition d'“intrus", étranger au fonctionnement interne du texte.

Dans cette tendance restauratrice du rôle du lecteur, Umberto Eco se signale par la radicalité de sa thèse centrale: "toute œuvre d'art est ouverte à un éventail illimité de lectures possibles." (idem: 156). L'œuvre moderne, notamment, est régie par un processus d'ouverture-clôture induisant une lecture créatrice (Eco, 1965: 18). De ce fait, on peut escompter pour un texte littéraire plusieurs lectures possibles.

Fondée sur la notion empruntée à l'esthétique de la réception de "lecteur implicite","prévu“ par le texte, selon laquelle "(...) le texte postule la coopération du lecteur comme condition d'actualisation" (Eco, 1985: 65), la thèse d'Eco présuppose un "lecteur modèle" dont l'auteur et le texte peuvent escompter une compétence de résolution d'énigmes ou de zones d'indétermination du texte (Compagnon, 1998: 164s), alors que, simultanément, ils instituent ou programment cette compétence (Eco, 1985: 68s).

Pareille démarche, présuppose que l'on accepte la norme du roman réaliste ou naturaliste du XIXe siècle dont le schéma narratif est complet. 
D'où les limites d'une telle approche du lecteur et que Compagnon ne manque pas de relever :

Mais que dire du lecteur qui n'a pas reçu cette initiation traditionnelle au roman ? Ou encore le roman contemporain, parfois qualifié de postmoderne, fragmentaire et déstructuré ? Sa conduite est-elle encore réglée par une quête de cohérence sur le modèle du roman réaliste ? (Compagnon, 1998: 164s).

De fait, nous voilà confrontés à la problématicité d'un éventail de lectures impliquant une compétence prévue par le texte et son auteur. Qui plus est, le caractère souvent ludique, ironique et citationnel, c'est-à-dire surcodé d'une certaine taxinomie du roman contemporain "postmoderne" français, du fait de son schéma narratif fragmentaire, juxtaposé, bâti sur des ruptures de ton et en clin d'œil vis-à-vis de l'héritage littéraire classique et moderne, met en jeu, pour reprendre Demoulin : “(...) un extérieur qui se veut littéraire, il ne s'adresse qu'à des déjà lecteurs." (Demoulin, 1997: 11).

Dominique Rabaté fait coïncider ce lecteur averti et complice avec le "lecteur implicite" et modèle. Selon lui, l'auto-référentialité postmoderne, particulière et parodique, thématise le lecteur, en fait un personnage, voire une série de personnages ; et "(...) réclame de lui une plus large compétence textuelle [mémoire des œuvres, ironie des codes]“ (Rabaté, 1998: 123).

En somme, le tournant opéré sur la fiction française contemporaine a aussi eu pour mérite de prendre conscience du rôle du lecteur dans le fait littéraire et d'en définir la portée après les mouvements textuels et théoriques (Nadaud, 1992: 77), même si les outils théoriques, une fois raisonnés par le sens commun, s'avèrent utiles.

Aussi Compagnon assigne-t-il un rôle équilibré, mais vital, à la lecture; pratique "double, ambiguë, déchirée" qu'il situe, à l'instar des autres activités humaines, "dans l'entredeux" (Compagnon, 1998: 175).

D'aucuns, encouragés par cette réhabilitation du lecteur, n'hésitent pas à anticiper sur un rôle encore imprévisible de ce dernier, mais décisif et actif dans le fonctionnement narratif du texte. En effet, "l'apparition de nouveaux supports d'écriture, liés aux développements de l'informatique et d'Internet" (Rabaté, 1998: 122) favorisent une coélaboration du texte et induisent une logique interactive entre les instances de l'auteur (intention) et du lecteur (réception).

II n'est pas avéré cependant que l'interactivité induite par des supports nouveaux, tels que l'hypertextualité finisse par supplanter la rencontre symbolique et vitale que s'obstinent à maintenir l'écriture et la lecture. D'autant plus, comme le rappelle Dominique Rabaté, que "l'essence de la littérature est précisément (...) d'agencer des mises en forme pour dire ce 
qui resterait indicible ou muet", tandis que la lecture "réarrange toujours le sens inépuisable du texte" et par la même occasion "réorganise sans cesse le rôle du lecteur" (Rabaté, 1999: 97).

À cet égard, le lumineux article de Nicolas Ancion dans Écritures s'avère une contribution originale et judicieuse à la réélaboration du rôle du lecteur dans le cadre du champ littéraire postmoderne. Basé sur le timide rapprochement de Roland Barthes et du lecteur opéré dans $S / Z$ ou Le Plaisir du texte, et sur les nuances notionnelles de "textelecture de plaisir","texte-lecture de jouissance“, ce critique dégage la nature changeante de la lecture dans son contexte contemporain.

Pour ce faire, il rappelle que la lecture littéraire se joue entre le texte et le lecteur, et implique un «protocole de lecture», pacte par lequel le lecteur assigne une fonction au texte étant donné "le but que le lecteur confère à sa lecture, et qui ressortit aussi bien à la nature du texte qu'à la compétence lectrice“ (Ancion, 1993: 30s).

L'infinitude de protocoles de lecture suggère leur regroupement en "modalités protocolaires" (idem: 32), un facteur socio-historique renvoyant à la consolidation des systèmes scolaires obligatoires et à l'émergence d'un champ littéraire autonome selon la conception bourdieusienne. Et Ancion d'énumérer les trois modalités protocolaires ayant cours durant la modernité littéraire.

D’abord, la lecture en tant que "plaisir", c'est-à-dire la reconnaissance et recréation du sens préexistant et indéfini du texte. La critique textuelle et les revues littéraires la pratiquent à l'envi. Ensuite, la lecture en tant que devoir, issue de l'institution de la scolarisation obligatoire. Enfin, la lecture en tant que "jouissance", à savoir celle qui attribue davantage d'importance à l'acte de lecture en soi qu'au texte lu. Cette modalité de lecture, une fois appliquée à la littérature, cherche dans un texte un discours neuf, non encore exprimé.

À ce propos, Roland Barthes avait pertinemment relevé ces nuances théoriques qu'il s'agit plus que jamais de confirmer dans le champ littéraire actuel. L'écrivain de plaisir et son lecteur relèvent d'un "parler sur" (Barthes, 1973: 37), où le langage se superpose à la parole, puisque "ce plaisir peut être dit : de là vient la critique" (idem: 82s). En revanche, l'écrivain de jouissance, et son lecteur, sont aux prises avec un "texte intenable","texte impossible" renvoyant à un "parler en" (idem: 37 ) fonctionnant "hors de toute finalité imaginable" (idem: 83).

Entre plaisir et jouissance, il est une "marge d'indécision", de glissement et d'oscillation où s'ouvre une zone d'indétermination, entre la prévisibilité du texte et le travail de lecture : "ce lecteur, il faut que je le cherche, [que je le 'drague'], sans savoir où il est.". Un espace de jouissance est alors créé : “Ce n'est pas la 'personne' de l'autre qui m’est 
nécessaire, c'est l'espace (...).“ (idem: 11). La jouissance réfère à cette atopie du texte et de sa lecture, en situation "hors-critique" et "hors-plaisir" (idem: 37).

Nicolas Ancion conclut que la lecture comme plaisir, le "parler sur" barthésien a largement dominé le discours littéraire moderne. Toutefois, il trahit de plus en plus les symptômes d'une crise dont on devine déjà quelques aspects : le franchissement d'un seuil critique et fatidique avec le Nouveau Roman et la textualité pour ce qui est du "plaisir" escompté à la lecture d'un texte; la déhiérarchisation des genres littéraires, et la relativisation de la légitimité littéraire des textes (leur rapport à la culture lettrée), ainsi que la réhabilitation intégrale du rôle créatif du lecteur.

Dès lors, la mutation en cours revendique, pour le contemporain littéraire, une liberté esthétique ainsi que l'affirmation des droits du lectorat au détriment des privilèges de l'auteur ou de ceux du texte. Il suffirait pour s'en convaincre de considérer l'impact de l'"effet Pivot" et la médiatisation événementielle de la littérature dans "Apostrophes"; laquelle, "dans une mobilité postmoderne" (Health, 1993: 1018), dissout les questions que le siècle se posait sur la nature et la fonction du phénomène littéraire : "Qu'est-ce que la littérature ?" et "Que peut la littérature ?".

Animée, non par un "spécialiste" de la littérature, mais bien par quelqu'un souhaitant partager le simple plaisir de la lecture, l'émission "Apostrophes", comme l'a remarqué Stephen Heath : “(...) n'a pas inspiré une littérature véritablement populaire, pas plus qu'il n'a ouvert de perspectives critiques permettant de comprendre les pratiques d'écritures contemporaines." (idem: 1019). Est-ce un tort ? À vrai dire, l'effet Pivot ne s'est jamais voulu de l'ordre du "parler sur", mais rejoint plutôt une jouissance lectrice en partage, au risque de niveler les romans et leurs auteurs : "sur un terrain égalisé à l'étalon de 'l'intéressant', il [Pivot] soumettait livres et auteurs, spectateurs et lecteurs au bonheur d'un échange sans nuage : on est entre proches - la télé, Pivot et nous." (ibidem).

Or, en France, deux textes, à des moments différents, sont venus baliser cette réflexion et cette mutation. Tout d'abord, le succès, dès sa parution en 1992, de Comme un roman de Daniel Pennac laisse entendre que cet essai, au titre subtilement provocateur, traduisait une réelle aspiration du lectorat à envisager l'acte de lecture sous l'angle de la liberté et de la jouissance, ainsi qu'une exaspération à l'endroit des modalités protocolaires de plaisir et de devoir dominant les pratiques modernes de lecture.

Deux idées fortes balisent ce livre. D'une part, la lecture devient de plus en plus incompatible avec le devoir et le dogme : "le verbe lire ne supporte pas l'impératif“" (Pennac, 1992: 13). D'autre part, le lecteur y met forcément du sien : "la lecture est un acte de création permanente“ (idem: 27).

Dès lors, Pennac inverse la logique du devoir de lecture et lui substitue la revendication de "droits" sous forme d'une charte postmoderne des droits imprescriptibles du 
lecteur qui agacerait plus d'un critique et plus d'un enseignant. Aussi le lecteur se voit-il reconnaître des prérogatives subvertissant l'approche moderne de la lecture : le droit de ne pas (vouloir) lire, le droit de sauter des pages ou de limiter sa lecture à la quatrième de couverture, le droit de commencer une lecture et de ne pas la finir, le droit de relire, le droit de lire n'importe quoi indépendamment des hiérarchies des genres littéraires ou des contraintes de la culture lettrée, le droit à l'identification (fût-elle naïve) avec les personnages de romans lus et d'y éprouver une "jouissance" toute particulière, le droit de lire n'importe où, le droit de lire ici et là en passant d'un livre à l'autre, de lire à haute voix ou encore de se taire (idem: 165-198).

Ainsi, plus qu'hésiter entre lecture de plaisir et lecture de jouissance, notre époque entend d'une part niveler l'importance et le prestige des modalités de lectures héritées de la modernité ; et d'autre part s'investir dans un éventail de pratiques de lecture axées sur la "jouissance", fût-ce au risque de se taire : "Grande jouissance de lecteur, ce silence d'après la lecture“ (idem: 20).

Par ailleurs, et plus récemment, l'essai de Pierre Bayard (2007) est venu complexifier la théorisation de (non)lecture en lui apportant une touche psychanalytique dont l'auteur avait déjà fait preuve à d'autres moments.

En fait, Bayard creuse l'intuition un peu ludique du protocole de lecture très élastique proposé par Pennac, et qui allait, rappelons-le, jusqu'au droit à ne pas lire. C'est à nouveau les contraintes protocolaires associées à la lecture qui sont interrogées ici : l'obligation de lire, l'obligation de tout lire, mais surtout "le discours tenu sur les livres" (idem: 14).

Pour une "nation littéraire" (Fergusson, 1991) comme la France, dont les liens sociaux sont quelque part liés au prestige et à la reconnaissance de l'écrivain et de la littérature, cette approche met à nu un malaise latent et une insécurité discursive qui frôle "la culpabilité inconsciente“ (idem: 15), voire une fraude du statut même de l'écrivain et de la littérature si l'on en croit François Bégaudeau (2008).

De ce point de vue, Bayard pointe une des conditions majeures de l'acte de lire, et qui chez Pennac n'osait pas encore dire son nom: la notion de "non-lecture", voire "délecture" en tant que dérogation à la modalité protocolaire basée sur le devoir, ne se substitue aucunement, purement et simplement, à la notion de lecture.

Au contraire, elle éclaire le phénomène singulier d'entre-deux qu'est la lecture étant donné le discours, social notamment, censé s'élaborer dans sa foulée ; le discours attendu socialement sur le fait littéraire, l'accomplissement d'un devoir engendrant une panoplie de stratégies, comme la construction d'autant d'effets de réels placés dans le discours.

Ce faisant, Pierre Bayard dresse un éventail d'incidents protocolaires de "lèselecture" qui ne peuvent que s'inscrire dans une modalité protocolaire autre, et en tous cas 
mise au ban de l'institution littéraire : "livre inconnu“, "livre évoqué", "livre oublié", "livre parcouru“; des catégories que Pennac n'aurait pas démenties.

Ces réalités littéraires, mais surtout subjectives, vécues dans le non-dit de la lecture, se placent dans l'entre-deux de l'expérience personnelle et collective. Elles opèrent une inversion de l'approche d'Umberto Eco sur l'instance réceptrice du texte littéraire. Le "lecteur implicite" fait place au "livre intérieur": "cet ensemble de représentations mythiques, collectives ou individuelles, qui s'interposent entre le lecteur et tout nouvel écrit, et qui façonnent la lecture à son insu“ (idem: 81).

Cette intuition, partagée par l'expérience lectrice de tout un chacun, renvoie à l'incommunicabilité même du vécu de la lecture, placé dans l'indéfinissable entre-deux du discours (ou de son exigence sociale et institutionnelle) et de la subjectivité. Le "livre intérieur" n'est ainsi "transmissible à personne et superposable à aucun autre" (idem: 94). Le livre subjectif s'avère dès lors toujours plus riche et aux implications plus profondes qu'il n'y paraît au premier abord. Le "livre-fantôme" (idem: 140) dont parle Pierre Bayard dessine, tout compte fait, des fantasmes et des non-dits qui engagent les cultures littéraires et leur perception dans le contexte présent.

C'est dire combien la culture littéraire est plus complexe que ce qu'on en dit par tâtonnements et bredouillements gênés çà et là. C'est le cas justement de la leçon inaugurale de Compagnon au Collège de France intitulée La littérature pour quoi faire? (2007). Notre propos ici n'est certes pas de rabattre les conclusions du théoricien, mais leurs généralités laissent pantois et risquent même d'apporter de l'eau au moulin de ceux pour qui la culture littéraire n'est d'aucune efficacité, n'implique aucun développement et n'est le moteur d'aucune performance dans le monde actuel ${ }^{1}$.

Oui, "Il y a donc une pensée de la littérature. La littérature est un exercice de pensée ; la lecture, une expérimentation des possibles“ (idem: 70). Certes, la lecture “(...) contribue donc de manière irremplaçable à l'éthique pratique comme à l'éthique spéculative" (idem: 62).

II y a plus à dégager quand on admet que le processus de lecture se trouve ancré dans l'immémorial du récit, dans sa version écrite; qu'elle en vient à être une donnée anthropologique incontournable, même si ses modalités sont changeantes et qui engagent l'apprentissage, l'agencement social, la convivialité, la communication pratique, l'expression, le politique, le sacré.

Bref, des assises anthropologiques et culturelles qui rendent la lecture non pas "irremplaçable", mais véritablement "incontournable".

\footnotetext{
${ }^{1}$ Le journal Le Monde rappelait, d'ailleurs, que "Là aussi, dans un entretien d'embauche, à qualification égale, on choisira celui qui est capable de se situer lui-même par rapport au monde" ( EUDES, Yves \& LARONCHE, Martine - "À quoi sert l'épreuve de philo ?", Le Monde, 14 juin 2006).
} 


\section{Bibliographie}

ANCION, Nicolas (1993). "La lecture littéraire, entre post- et modernité". In: Écritures, no 5, Le dépli. Littérature et postmodernité.

BARTHES, Roland (1973). Le plaisir du texte. Paris: Seuil.

BAYARD, PIERRE (2007). Comment parler des livres qu'on n'a pas lus ? Paris: Minuit.

Begaudeau, François (2008). Antimanuel de littérature. Paris: Breal.

CALINESCU, Matei (1999). As 5 faces da modernidade. Lisboa: Vega.

COMPAGNON, Antoine (1998). Le démon de la théorie. Littérature et sens commun. Paris: Seuil.

COMPAGNON, Antoine (2007). La littérature pour quoi faire ?. Paris: Fayard.

Demoulin, Laurent (1997). "Génération innommable". In: Textyles, no 14, Lettres du jour (II).

Eco, Umberto (1965). L'œuvre ouverte. Paris: Seuil.

Eco, Umberto (1985). Lector in fabula. Le rôle du lecteur. Paris: Grasset.

FERGuSON, Priscilla Parkhurst (1991). La France, nation littéraire. Bruxelles: Labor.

GUICHARD, Thierry (1995). "Le roman en question II". In: Prétexte, no 8.

HEATH, Stephen (1993). "Les causeries du vendredi”. In: De la littérature française. Paris: Bordas.

NADAUD, Alain (1992). Malaise dans la littérature. Paris : Champ Vallon.

PENNAC, Daniel (1992). Comme un roman. Paris: Gallimard.

RABATE, Dominique (1998). Le roman français depuis 1900. Paris: PUF, "Que sais-je ?".

RABATE, Dominique (1999). "États de la prose contemporaine". In : Prétextes, n²1/22, pp. 91-97. 